\title{
Challenging the osseous component of sphenoorbital meningiomas
}

\author{
Svenja Maschke ${ }^{1}$ Mauricio Martínez-Moreno ${ }^{1} \cdot$ Alexander Micko $^{1} \cdot$ Matthias Millesi $^{1} \cdot$ Georgi Minchev $^{1}$. \\ Ammar Mallouhi $^{2} \cdot$ Engelbert Knosp $^{1} \cdot$ Stefan Wolfsberger ${ }^{1}$
}

Received: 8 June 2019 / Accepted: 12 July 2019/Published online: 1 August 2019

(C) The Author(s) 2019

\begin{abstract}
Background Intraosseous growth is a unique feature of sphenoorbital meningiomas (SOM). Its close relation to neurovascular structures limits complete surgical resection and possibly contributes to the high recurrence rate.

Objective To evaluate the growth behavior of intraosseous remnants and develop a protocol for precise intraoperative visualization of intraosseous SOM.

Methods We included 31 patients operated for SOM from 2004 to 2017. The growth velocity of the intraosseous tumor component was volumetrically calculated in 20 cases. To improve accuracy of image guidance, we implemented a specialized bone surface-based registration algorithm. For intraoperative bone visualization, we included CT in multimodality continuous image guidance in 23 patients. The extent of resection (EOR) was compared with a standard MR-only navigation group $(n=8)$. Results In 11/20 cases (55\%), a progressive regrowth of the intraosseous SOM remnant was noted during a mean follow-up of 52 months (range 20-132 months). We observed a mean increase of $6.2 \mathrm{~cm}^{3}$ (range $0.2-23.7 \mathrm{~cm}^{3}$ ) per patient and side during the follow-up period. Bone surface-based registration was significantly more accurate than skin surface-based registration (mean 0.7 $\pm 0.4 \mathrm{~mm}$ and $1.9 \pm 0.7 \mathrm{~mm}, p<0.001)$. The EOR of the intraosseous component was significantly higher using CT + MRI navigation compared with controls ( $96 \%$ vs. $81 \%, p=0.044)$.

Conclusion Quantitative assessment of the biological behavior of intraosseous remnants revealed a continuous slow growth rate independent of the soft tumor component of more than half of SOM. According to our data, application of a multimodal image guidance provided high accuracy and significantly increased the resection rate of the intraosseous component of SOM.
\end{abstract}

Keywords Bone infiltration $\cdot$ Image guidance $\cdot$ Meningioma $\cdot$ Skull base $\cdot$ Sphenoorbital meningioma

\section{Introduction}

Apart from their dural component, sphenoorbital meningiomas (SOM) exhibit a unique intraosseous growth pattern within and adjacent to the sphenoid bone closely related to skull base neurovascular structures limiting surgical resection.

This article is part of the Topical Collection on Tumor - Meningioma

Stefan Wolfsberger

stefan.wolfsberger@meduniwien.ac.at

1 Department of Neurosurgery, Medical University of Vienna, Waehringer Guertel 18-20, 1080 Vienna, Austria

2 Department of Biomedical Imaging and Image-guided Therapy, Medical University of Vienna, Waehringer Guertel 18-20, 1080 Vienna, Austria
With up to $9 \%$ of all adult intracranial meningiomas [1,2], SOM are not infrequent, and the vast majority is graded as WHO ${ }^{\circ} \mathrm{I}(84-100 \%)$ [3-5].

Tumor extension into the orbit results in the most common initial complaints of proptosis (86-93\%) [5-7] and visual impairment (65-78\%) [5, 6]; extension into the middle cranial fossa, cavernous sinus, and infratemporal fossa may cause later compressive symptoms.

The main goals of neurosurgical SOM treatment are improvement/prevention of visual impairment and reversal of exophthalmos by decompression of optochiasmatic and orbital structures from affected bone and soft tumor component. Albeit the intradural SOM being mostly amenable to extensive surgical removal, the dural involvement of orbital apex, superior orbital fissure (SOF), and cavernous sinus remains challenging. Concerning the intraosseous component of SOM, extensions medial to the cranial nerve ostia of the middle cranial fossa render a Simpson ${ }^{\circ} \mathrm{I}$ resection virtually impossible from a standard pterional approach. These growth 
characteristics contribute to the high recurrence rates of SOM after primary surgery, amounting to up to $60 \%$ after 5 years [8-11].

In the case of pure sphenoid wing meningiomas, recurrence rates were reported higher if bone infiltration was present (> $30 \%$ vs. $11.6 \%$, respectively) [12]. In line with Simpson's observations, a complete resection of the involved bone has been advocated to prolong progression-free survival in these meningiomas $[2,5,13-15]$. Regarded as a subtype of sphenoid wing meningiomas, this may also apply for SOMs.

Remnants of infiltrated bone are common after SOM surgery; however, the growth potential of the intraosseous SOM remnant remains unclear as it has not been systematically analyzed to date.

Microsurgical resection of osseous tumor extensions is impeded by the difficulty to visually distinguish osseous tumor from surrounding healthy bone intraoperatively. However, the extent of altered bone can easily be recognized in bonewindowed CT images.

The aims of the present study were (1) to evaluate the potential of regrowth of intraosseous remnants of SOM after subtotal resection, (2) to develop a protocol for precise intraoperative visualization of the intraosseous SOM component in relation to adjacent neurovascular structures, and (3) to assess whether the extent of resection (EOR) can be safely increased by the proposed method.

\section{Patients and methods}

The study cohort comprises 31 patients with primary surgery for SOM between 2004 and 2017 in a tertiary care institution, i.e., $3.1 \%$ of all 1041 surgically treated intracranial meningiomas during this period.

Only cases with an attempted GTR were included. Planned partial resections, meningiomas located mainly beyond the confines of the sphenoorbital region, optic nerve sheath meningiomas, and meningiomatoses were excluded.

This study was approved by the ethics committee (EC no: 1144/2019).

\section{Patient and tumor characteristics}

Clinical presentation: the presenting symptoms were extracted from the clinical information system, and patients were invited for follow-up interviews. Proptosis was assessed on preoperative $\mathrm{CT}$ images.

Histopathologic evaluation: the intraosseous tumor component was evaluated for meningioma infiltration. Meningioma specimens were examined for their histopathological type and WHO grade [16-18].

\section{Intraosseous growth assessment}

CT scans are required to assess the extension of the osseous SOM component. All patients received CT scans within $48 \mathrm{~h}$ postoperatively and within the follow-up clinical examination of this study (additionally to the routine MRI follow-up examinations). As radiologic follow-up is usually performed by MRI that does not provide sufficient information on the evolution of intraosseous remnants, patients with a follow-up of $\geq 1$ year were invited for CT scans.

An identical CT scanner and protocol was used for all scans (Siemens Somatom Sensation 64®, $0^{\circ}$ gantry tilt, 1-mm spiral distance, $120 \mathrm{kV}, 380 \mathrm{~mA}$ ). Image manipulations and navigation guidance were all performed with a StealthStation S7 System (Medtronic, CO, USA). To determine the growth behavior of the intraosseous tumor component, the bone infiltration of the anterior and middle cranial fossa was first segmented semiautomatically. Then, the tumor volume was compared

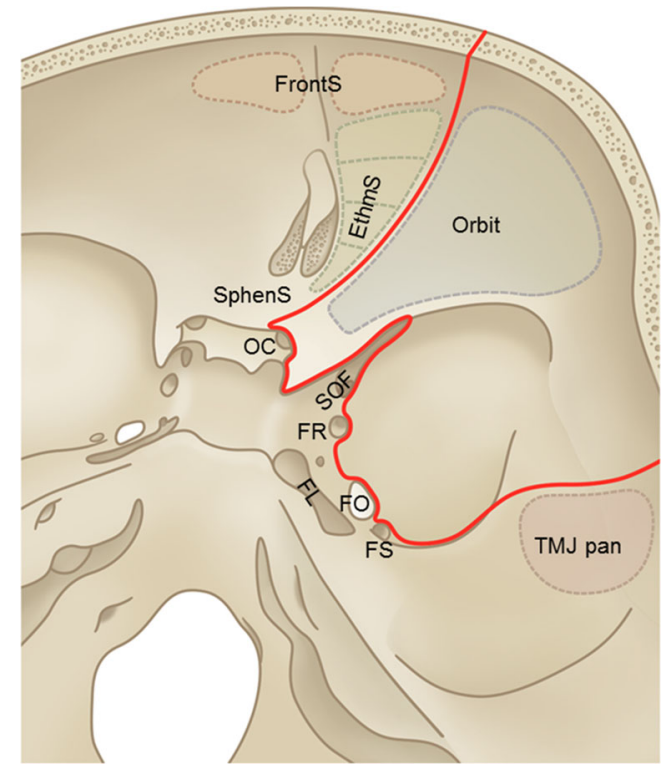

Fig. 1 Neurovascular limit for safe resection of the intraosseous component of SOM. To safely accomplish removal of the largest part of the intraosseous tumor component, we propose an anatomical limit based on critical neurovascular structures. From a standard pterional approach, this limit was defined by the frontal, ethmoid, and sphenoid sinuses (FrontS, EthmS, SphenS), the medial wall of the optic canal (OC), superior orbital fissure (SOF), foramen rotundum (FR), foramen ovale (FO), foramen lacerum (FL), and temporomandibular joint (TMJ) pan. FS $=$ foramen spinosum 
and the growth velocity calculated between early postoperative and follow-up CT scans.

\section{Definition of anatomical limits for safe resection}

To prevent neurovascular injury and sinunasal repair during a pterional approach, we propose anatomical limits to safely accomplish maximum SOM removal (Fig. 1):

for the intraosseous SOM component, this resection limit was defined medially by ethmoid and sphenoid sinuses, medial wall of the optic canal, SOF, foramina rotundum, ovale and lacerum, and the temporomandibular joint pan.

The soft tumor resection was limited by the contents of the SOF, orbital apex structures (annulus of Zinn), and cavernous sinus structures.

\section{Improvement of image guidance}

\section{Registration accuracy}

A specialized bone surface-based registration algorithm was implemented to improve accuracy of image guidance for surgery of the osseous SOM component: preoperatively, a 3D skull surface model was created by threshold segmentation from CT data. After elevation of the skin flap, this model was registered to the exposed fronto-temporo-zygomatic bone surface, comparable with skin surface-based registration. Registration accuracy was checked on anatomical landmarks before proceeding with surgery (Fig. 2).

Prior to translation into the clinical setting, we compared feasibility and accuracy of this novel bone surface registration in a cadaver experiment with standard skin surface and bone screw registration.

\section{Image guidance protocol}

Bone-windowed CT scan To improve visualization of the osseous SOM component, we merged a bone-windowed CT scan onto the routine contrast-enhanced T1-weighted MR (CE-T1MR).

Preoperatively, we semiautomatically segmented the osseous SOM component on the planning software. To facilitate identification of the infiltrated bone during surgery, the contours of the intraosseous SOM component were displayed in multiplanar views intraoperatively.

For higher accuracy, this CT scan (see imaging parameters above) was selected as registration reference [19].

Real-time tracking To improve safety of removal of the osseous SOM component in the vicinity of neurovascular structures, we included real-time instrument tracking that provided continuous update of the resection progress utilizing instruments such as a navigated drill or tip-tracked suction device as described previously [19].

\section{Group assignment}

All 31 patients were operated by five experienced skull base surgeons. The intent of surgery was always the maximum removal of both soft and osseous SOM components. The impact of the addition of bone-windowed CT scan to MR image guidance (group CT $+\mathrm{MRI}, n=23$ ) on the extent of resection was compared with MRI-only navigation (group
Fig. 2 Bone surface-based registration: 3D-model used for registration (a). Concurrent intraoperative view of the exposed bone (b) with attached bone-attached patient reference tracker and landmark check of the coronal suture after bone surfacebased registration $(\mathbf{c})$






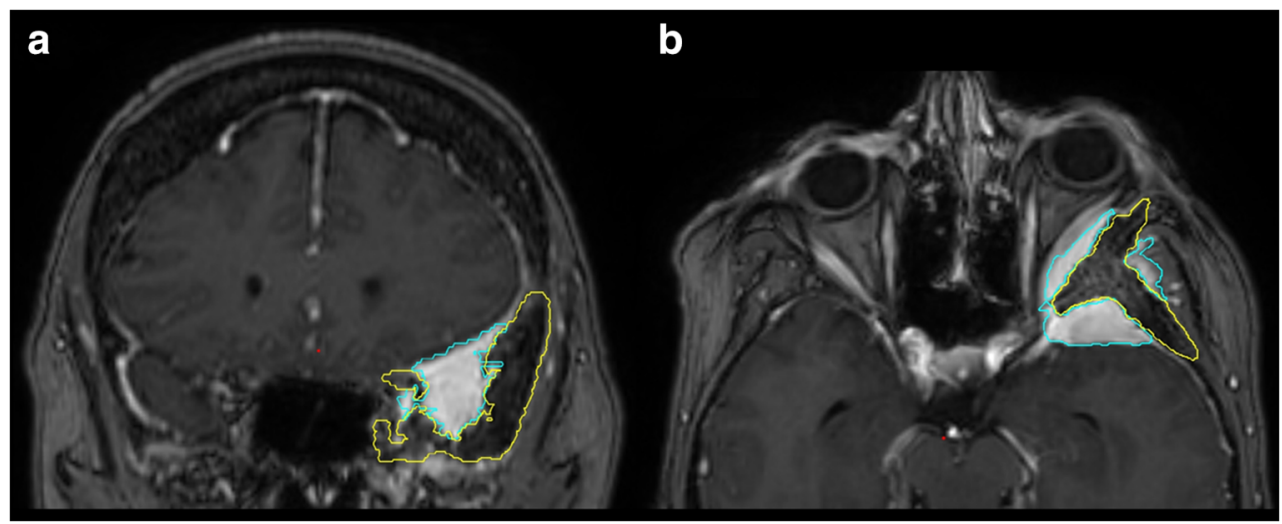

Fig. 3 Case 49f, left-sided SOM: intraoperative multimodality image guidance concurrently displaying (1) osseous tumor extension (CT-based segmentation, yellow) and (2) soft tumor extension (MR T1 CE-based, blue). The background image is a MR T1 CE fat suppression MRI. a Soft tumor extension into the orbit via the SOF with surrounding osseous
SOM involvement of the anterior clinoid process, the middle fossa floor extending up to the lateral wall of the sphenoid sinus, and the temporal bone. b Soft tumor extensions at the temporopolar dura, the SOF, the lateral orbit, and the temporal muscle. Osseous tumor extension in the greater wing of the sphenoid bone
MRI, $n=8$ ). The choice of the image guidance protocol was not based on tumor volume or extension but only on individual preference.

\section{Extent of resection analysis}

Areas of osseous tumor involvement were defined pre- and postoperatively on CT-based volumetric studies. Tumor borders were segmented semiautomatically, and the tumor volume $\left(\mathrm{cm}^{3}\right)$ was calculated. The soft tissue component of the SOM was identified separately in preoperative contrastenhanced T1-weighted MRI fat suppression sequences (CET1MRI) in the same manner for all patients and postoperatively in eight patients who provided an MRI at follow-up (Fig. 3).
Residual tumor volumes of the intraosseous component (deemed resectable according to the line in Fig. 1) and of the soft tumor component were compared with preoperative tumor volumes in order to determine the EOR (Fig. 4).

\section{Statistical analysis}

Variables are described as mean or median with range as appropriate. Mann-Whitney $U$ test for differences in not normally distributed independent parameters between study and control group was applied. Asymptotic significances were chosen as two-tailed $p$ values.

$p$ values $<0.05$ were considered significant. Analyses were carried out using SPSS software version 22.0 (IBM Corporation, USA).
Fig. 4 Operative result of case $54 \mathrm{f}$ by merging preoperative (yellow) and postoperative (orange) CT scans. a The osseous tumor remnants (orange) are mainly confined medial to the proposed line. b Removal of the lateral parts of the greater sphenoid wing. Resection was limited by involvement of the foramen rotundum and lateral sphenoid sinus wall. c Removal of the middle cranial fossa infiltrate lateral to the oval foramen. d Osseous remnant covering the TMJ pan medially and anteriorly

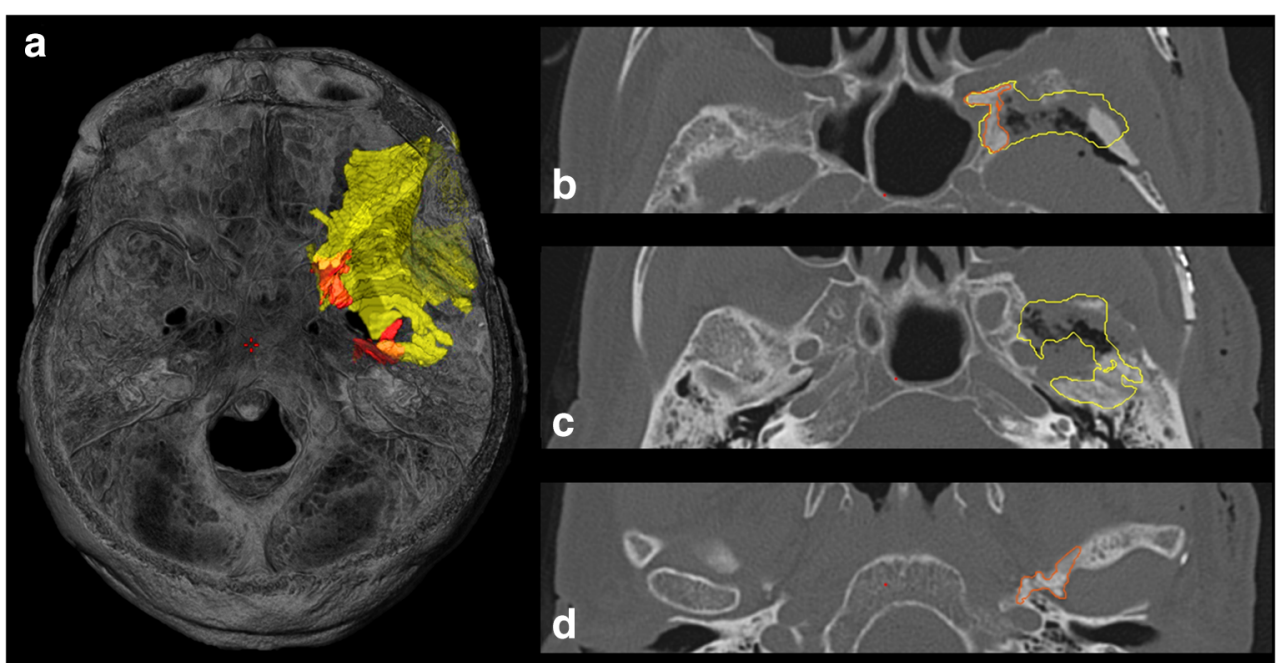


Table 1 Patient and tumor characteristics

\begin{tabular}{|c|c|c|}
\hline Patient characteristics & $n$ & $(\%)$ \\
\hline No. of patients & 31 & \\
\hline Age (median, range) & $51(38-82)$ & \\
\hline \multicolumn{3}{|l|}{ Sex } \\
\hline Female & 27 & $(87.7 \%)$ \\
\hline Male & 4 & $(12.9 \%)$ \\
\hline $\mathrm{M}: \mathrm{F}$ ratio & $1: 6.75$ & \\
\hline \multicolumn{3}{|l|}{ Side } \\
\hline Right & 15 & $(48.8 \%)$ \\
\hline Left & 13 & $(41.6 \%)$ \\
\hline Bilateral & 3 & $(9.6 \%)$ \\
\hline \multicolumn{3}{|l|}{ Histopathological type } \\
\hline Meningothelial ${ }^{\circ} \mathrm{I}$ & 22 & $(71.0 \%)$ \\
\hline Secretory ${ }^{\circ} \mathrm{I}$ & 5 & $(16.1 \%)$ \\
\hline Transitional ${ }^{\circ} \mathrm{I}$ & 1 & $(3.2 \%)$ \\
\hline Clear-cell ${ }^{\circ} \mathrm{II}$ & 2 & $(6.4 \%)$ \\
\hline Anaplastic ${ }^{\circ} \mathrm{III}$ & 1 & $(3.2 \%)$ \\
\hline \multicolumn{3}{|l|}{ Presenting symptoms } \\
\hline Loss of visual acuity & 14 & $(45.2 \%)$ \\
\hline Temporal or lid swelling & 8 & $(25.6 \%)$ \\
\hline Lacrimation & 6 & $(19.2 \%)$ \\
\hline Headaches & 5 & $(16 \%)$ \\
\hline Conjunctival injection & 5 & $(16 \%)$ \\
\hline Double vision & 4 & $(12.8 \%)$ \\
\hline Ptosis & 2 & $(6.4 \%)$ \\
\hline Photopsia & 1 & $(3.2 \%)$ \\
\hline
\end{tabular}

\section{Results}

\section{Clinical presentation and tumor characteristics (Table 1)}

Patients presented average 14 months after onset of initial complaints (range $0-84$ months).

The most common presenting symptom in 27/31 (87\%) patients was unilateral proptosis of mean $5.1 \mathrm{~mm}$ (range $1.4-8.5 \mathrm{~mm}$ ) over the unaffected side (in the 28 cases of one-sided SOM) or $5.8 \mathrm{~mm}$ (range 4.4-7.8 $\mathrm{mm}$ ) over the less affected side (in the three cases of bilateral SOM).

Decline of visual acuity was reported by $14(45 \%)$ cases.

Surgical removal: all SOM resections were performed by standard microsurgical technique including high-speed diamond drill under neuronavigational guidance by senior surgeons.

For dural reconstruction, we used periosteum and/or synthetic dura substitute, for periorbital reconstruction fibrincoated collagen fleece. Osseous defects were reconstructed with polymethylmethacrylate bone cement. Cosmetic results were satisfactory except for one case of postoperative enophthalmos.

Histopathological findings: all but three SOMs were classified as $\mathrm{WHO}{ }^{\circ} \mathrm{I}$ - two were a clear-cell $\mathrm{WHO}{ }^{\circ} \mathrm{II}$ and the other one an anaplastic WHO ${ }^{\circ} \mathrm{III}$ meningioma.

Meningioma infiltration was confirmed in the intraosseous SOM component in all patients (31/31).

Adjuvant treatments: the three cases of WHO ${ }^{\circ} \mathrm{II}$ and ${ }^{\circ} \mathrm{III}$ received postoperative radiation therapy. Six patients with WHO ${ }^{\circ} \mathrm{I}$ and progressive disease after reoperations received radiation therapy $(n=4)$ or radiosurgery $(n=2)$.

\section{Growth behavior of the intraosseous SOM component (Fig. 5)}

For assessment of growth behavior of the intraosseous tumor component, our series comprised 25 patients with a minimum follow-up of 1 year. Of those, a follow-up CT scan was performed in 20 patients in the scope of this study. Of the remaining 5 patients, 3 were deceased (one of pancreatic cancer and two of unknown causes, including the patient with the WHO ${ }^{\circ}$ III meningioma) and 2 were unavailable.

During a follow-up of mean 52 months (range 20132 months), progression of the intraosseous remnant was noted in 11/20 cases $(55 \%)$ and an increase in volume of $6.2 \mathrm{~cm}^{3}$ (range $0.2-23.7 \mathrm{~cm}^{3}$ ) per patient and side; all but one atypical meningioma were graded as $\mathrm{WHO}{ }^{\circ} \mathrm{I}$. Hence, growth trend of intraosseous SOM was mean $1.48 \mathrm{~cm}^{3} /$ year (range $0.1-6.4 \mathrm{~cm}^{3} /$ year). All three bilateral cases showed progression of tumor remnants as well as of the untreated side (Fig. 5).

In $9 / 20$ cases (45\%), no regrowth of intraosseous remnants was found during the mean follow-up of 56 months (range 20-174 months). Of these patients except for one WHO ${ }^{\circ} \mathrm{II}$ meningioma, all patients had $\mathrm{WHO}^{\circ} \mathrm{I}$ meningioma.

Of note, the mean follow-up did not differ significantly between regrowth and non-regrowth cases.

\section{Results of the image guidance protocol}

\section{Feasibility}

Bone registration: in the lab setting, bone surface registration was significantly more accurate than skin surface registration (mean $0.7 \pm 0.4 \mathrm{~mm}$ and $1.9 \pm 0.7 \mathrm{~mm}$, respectively, $p<0.001)$ approaching the submillimetric accuracy of skull screw registration (mean $0.3 \pm 0.1 \mathrm{~mm}$ ).

Clinically, the bone surface registration was feasible in all cases providing high accuracy and special usefulness during osseous tumor drilling. In two cases of limited exposure of the zygomatic arch, registration was successful at second repetition. 


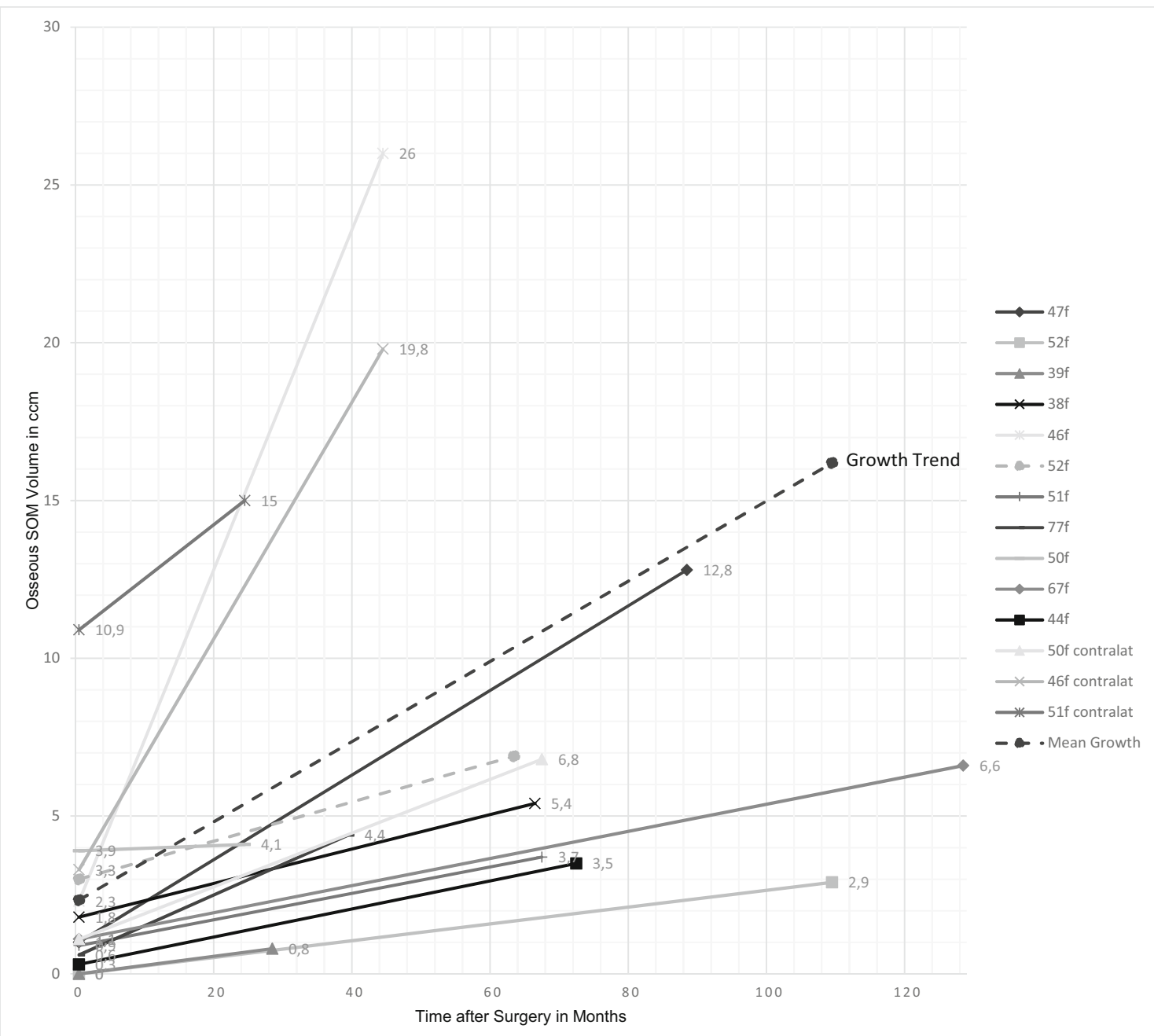

Fig. 5 Growth rate of the intraosseous tumor component of SOM: in 11/ 20 cases $(55 \%)$ with postoperative remnants and in all contralateral sides of the 3 bilateral tumors, a progressive regrowth was noted. Follow-up mean 52 months (range 20-132 months). Growth velocity mean $1.48 \mathrm{~cm}^{3}$ tumor increase per year (range $0.1-6.4 \mathrm{~cm}^{3} /$ year), indicated by the interrupted line

\section{Extent of resection analysis (Table 2, Fig. 6)}

Of all 31 patients, 23 (74\%) were operated with the image guidance protocol; the remaining $8(26 \%)$ operated with MR-only navigation served as controls. There were no statistically significant differences in preoperative tumor volumes of both the intraosseous $(p=0.206)$ and the soft tumor component $(p=0.414)$ between both groups.

For the intraosseous tumor component, EOR was significantly higher using the navigation protocol compared with MR-only navigation ( $96 \%$ vs. $81 \%$ respectively, $p=0.044$ ), but not for the soft tumor component.

We did not encounter increased operation times (mean duration 5.49 vs. $5.86 \mathrm{~h}$ for the multimodal image guidance vs. the MR-only navigation; $p=0.597$ ), indicating a similar setup time.

\section{Perioperative complications}

There was one case of new postoperative unilateral amaurosis due to optic nerve compression by hemostyptic material irreversible despite emergency revision. We observed two transient and one permanent oculomotor and one transient trochlear nerve dysfunctions. Transient hypesthesia was reported for V1 in two, V2 in four, and V3 in one of the cases and permanent hypesthesia for V1 and V2 in two cases each. Permanent xerophthalmia by lacrimal gland/nerve dysfunction was reported by five patients. 
Table 2 Extent of resection analysis

\begin{tabular}{lllll}
\hline Tumor volumes (ccm) & Group CT + MRI & Group MIR & $p$ value & $r$ (Dohen) \\
\hline Soft & & & & \\
$\quad$ Preoperative & $12.57 \pm 29.1$ & $7.81 \pm 4.08$ & 0.414 & 0.14 \\
$\quad$ Postoperative & $0.58 \pm 1.18$ & $2.05 \pm 2.05$ & 0.222 & 0.27 \\
$\quad$ Resected & $5.08 \pm 4.41$ & $6.50 \pm 0.98$ & 0.667 & 0.10 \\
Intraosseous & & & & \\
$\quad$ Preoperative & $16.14 \pm 8.80$ & $19.41 \pm 9.91$ & 0.206 & 0.22 \\
Postoperative & $0.67 \pm 1.04$ & $3.57 \pm 3.84$ & 0.029 & 0.39 \\
$\quad$ Resected & $15.46 \pm 8.68$ & $15.83 \pm 8.23$ & 0.542 & 0.10 \\
Total & & & & \\
$\quad$ Preoperative & $28.64 \pm 29.22$ & $31.15 \pm 10.83$ & 0.18 & 0.24 \\
Postoperative & $1.77 \pm 1.44$ & $6.00 \pm 5.09$ & 0.222 & 0.26 \\
Resected & $24.7 \pm 15.58$ & $31.05 \pm 6.15$ & 0.500 & 0.10 \\
EOR (\%) & & & & \\
Soft tumor & $96.11 \%$ & $78.95 \%$ & 0.286 & 0.25 \\
Intraosseous tumor & $95.80 \%$ & $81.09 \%$ & & 0.36 \\
Total tumor & $88.46 \%$ & $85.21 \%$ & 0.500 & 0.15 \\
\hline
\end{tabular}

We did not observe a significant increase in complication rate in the $\mathrm{CT}+\mathrm{MR}$ group despite a more aggressive bone removal.

\section{Discussion}

The growth potential of intraosseous remnants after incomplete SOM resection has not been systematically analyzed to date. As our data indicates a slow annual growth rate, maximum safe reduction of intraosseous SOM is warranted.

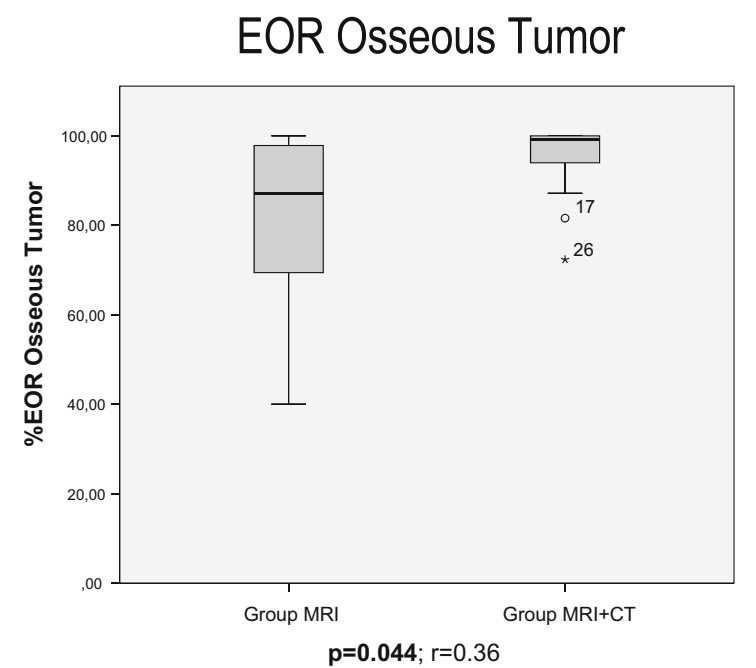

We propose an anatomical limit to which SOM can be safely resected. Further, we tested a novel image guidance protocol that was found to significantly increase the resection rate of intraosseous SOM.

\section{Osseous growth pattern}

In the case of skull base meningioma with sphenoid wing origin, large intracranial tumors with some hyperostotic changes are regarded a distinct feature (>90\%) [20] and suggest osseous tumor invasion by infiltration of the Haversian

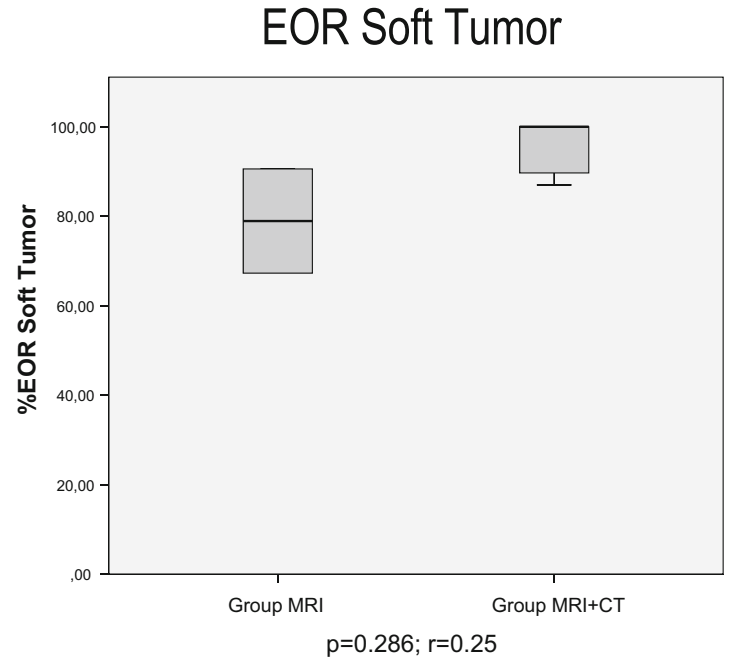

Fig. 6 Comparison of EOR between CT + MRI and the MRI-only group. EOR was significantly higher for the osseous tumor component $(p=0.044 ; r=$ $0.36)$, but not for the soft tumor component $(p=0.286 ; r=0.25)$ after application of the multimodality navigation protocol. 
system as first suggested by Echlin in 1934 [21, 22]. It still remains elusive to which extent other factors such as neoplastic enzymatic activity, osteoblastic stimulation by humoral factors, or vascular disturbances are involved [23-28]. The higher rate of recurrence after resection without the complete hyperostotic bone (Simpson $\geq$ II) supports such osseous meningioma infiltration $[6,14,29,30]$.

In the case of sphenoorbital meningiomas, the extensive sphenoid osseous changes are the distinct feature [31]. In the case of absence of the dural component, other hyperostotic conditions such as primary intraosseous meningioma, fibrous dysplasia, Paget's disease, and osteoma should be considered $[30,32]$.

The general hypothesis of SOM evolution is a primary dural origin with secondary osseous invasion [3]. There is a typical intradural temporopolar and periorbital growth of various globular sizes with possible en plaque extensions to the orbital roof and cavernous sinus. From the underlying intraosseous component, SOM may extend via sphenoid bone surfaces into temporal muscle and muscles of the infratemporal fossa or paranasal sinus mucosa as soft meningioma tissue. From these observations, a continuous growth pattern from dura via bone into extracranial soft tissues can be considered, but a primary sphenoid bone origin has not been ruled out.

Although there is no relation between the size of the tumor and the degree of hyperostosis $[8,31]$, the latter theory is supported by the observation that the intraosseous component can be disproportionately greater compared with the soft tumor component. This was reflected in our series with soft tumor volumes of mean $6.6 \mathrm{~cm}^{3}$ and osseous tumor volumes of mean $15 \mathrm{~cm}^{3}$.

Of note, the magnitude of the osseous invasion does not correlate with a more aggressive biological behavior [8].

Bilateral SOM are uncommon, yet three of our patients were found to have intraosseous and en plaque tumor components on both sides. Luetjens et al. proposed a staged resection with the more severely affected side treated first [33]. In our cases, only symptomatic sides and sides that increase in size were resected.

Our study shows that osseous remnants exhibit slow growth over the course of decades, and long-term follow-up is necessary for detection of clinical deterioration. One of our patients (not included due to lack of CT imaging studies in 1970s) with a 40+ year history of SOM developed a progressive exophthalmus and visual disturbances due to intraosseous tumor enlargement in the greater sphenoid wing with incipient narrowing of the orbital apex and was operated for recurrent SOM lately.

Therefore, maximum resection of the intraosseous SOM is warranted during primary surgery to prevent later inoperable infiltration of the skull base. This was highlighted by one case of our study that showed subsequent involvement of the frontal, ethmoidal, sphenoidal, and maxillary sinuses originating from a small postoperative remnant lateral to the proposed limit (Fig. 7).

In the cases of incomplete resection with osseous remnants, we suggest radiographic surveillance to early identify progression and potential soft tumor recurrence from these locations. The treatment armamentarium including reoperation, radiotherapy, and radiosurgery has to be individually tailored.

\section{Intraoperative image guidance}

Intraoperatively, the osseous component of SOM is difficult to visually distinguish from healthy bone and does not respect osseous sutures. As it can be readily identified on bonewindowed $\mathrm{CT}$, the inclusion of $\mathrm{CT}$ studies in image guidance to identify the intraosseous components has been proposed previously [34].

We advanced this concept by preoperatively segmenting intraosseous tumor areas on bone-windowed CT and intraoperatively displaying bony tumor extensions as colorized contours on the navigation screen.

Further, we applied tip-tracking of either drill or suction device as previously described [19], which allowed a seamless integration of navigation into the surgical workflow. Continuous instrument tracking was found useful in providing continuous information on the distance to neurovascular structures and osseous tumor borders. With the image guidance protocol, we were able to improve the EOR of the intraosseous component by $14 \%$.

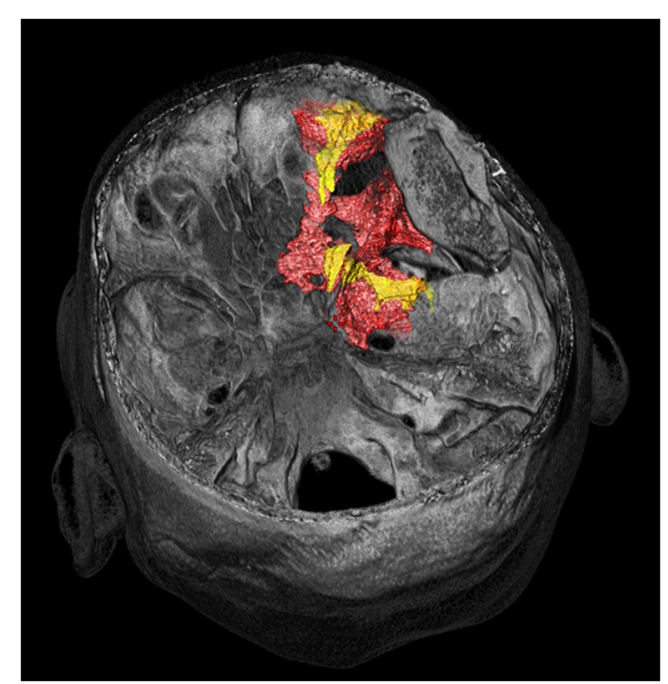

Fig. 7 Example for regrowth of osseous SOM remnant. Case 47f, meningothelial SOM (WHO I): right-sided SOM presenting with exophthalmus and visual disturbance. At initial presentation, the intraosseous meningioma component was confined to lateral of the proposed resection line. A complete resection of the soft component and a subtotal resection of the osseous component with restitution of exophthalmus and vision was achieved (yellow = remaining osseous tumor, $1.1 \mathrm{ccm})$. 
The duration of the resection was comparable between the study group and controls (5.4 h vs. $5.8 \mathrm{~h} ; p=0.59$ ), while more tumor volume was resected with the image guidance protocol.

\section{Bone surface-based registration}

The proximity of the intraosseous SOM component to neurovascular structures requires high navigational accuracy. In this study, we propose a bone surface-based registration as a highly accurate alternative to invasive bone screwbased $[35,36]$ registration or less accurate skin surfacebased registration that has not been applied for surgery of SOM before.

For lateral otorhinolaryngologic skull base surgery with retroauricular bone exposure, surface matching of the temporal bone and mastoid has been proposed by Zhou et al. [37].

After ex vivo tests, we could show that this registration method is feasible for SOM resections and can be easily incorporated into the surgical workflow. Registration of the exposed skull before craniotomy averts loss of neuronavigational accuracy from positioning of the patient, draping and attachment of retractors [36]. SOM resections are especially suited for bone surface-based registration due to exposure of three dimensionally traceable structures mainly of the zygomatic bone.

\section{Anticipation of critical skull base structures}

Visualization of modified bone may tempt the surgeon to extensive drilling, risking higher morbidity. We have therefore defined a resection margin at the skull base with medial limits at the cranial nerve foramina and ICA. Using continuous instrument navigation, the surgeon can anticipate these critical structures and duly limit tumor removal to avoid neurovascular injury.

Limited resection was also reported by other authors mainly inside the confines of the orbit around the SOF and the cavernous sinus [5, 38-41]. Adjuvant (stereotactic) radiation treatment/surgery has been applied in subtotally resected skull base meningiomas to control residual tumor and prolong progression-free survival $[42,43]$. In the cases of extensive skull base involvement, however, our proposed protocol has the potential to further increase resection rates.

\section{Vascular Complications}

Skull base surgery is fraught with injury to neurovascular structures. The risk of ICA injury can be minimized by anticipating the distance to the lacerum segment during drilling.

The ICA can be readily identified on CE-T1MRI, and CTA/MRA studies were not routinely included in the navigation protocol, but we recommend additional color coding of angiographic studies in selected cases with extensive bone infiltration of the middle cranial fossa floor to improve visualization of vascular structures and to avoid injury. The addition of angiographic images further aids in locating the middle meningeal artery for early tumor devascularization. In our series, no intraoperative ICA injuries were noted.

\section{Limitations of the study}

Study design Due to the rare incidence of SOM, the study design was retrospective. Due to the slow growing nature of the intraosseous SOM component, a more extensive followup duration of decades would be required for analysis of recurrence rates $[11,44]$.

Assessment of tumor growth Intraosseous SOM growth was calculated from only two time points, subtracting early postoperative CT scan remnant volume from a recent $\mathrm{CT}$ scan acquired within the scope of this study. Since no assumptions can be made about individual growth curves from two time points alone, it is only a crude estimate to display the growth trend and not representative for volumetric extrapolation [45].

\section{Conclusion}

Incomplete resection of the intraosseous component of sphenoorbital meningiomas is not infrequent. This first quantitative assessment of the biological behavior of intraosseous remnants revealed a continuous slow growth in more than half of the cases independent of the soft tumor component. Therefore, maximum safe reduction of the osseous tumor component is warranted but impeded by the proximity to neurovascular structures.

According to our data, addition of bone-windowed CT scan to image guidance was found to significantly increase the resection rate of the intraosseous component of sphenoorbital meningiomas.

Acknowledgments The authors would like to thank Mrs. Ingrid Dobsak, who provided us with Fig. 1.

Funding Information Open access funding provided by Medical University of Vienna.

\section{Compliance with ethical standards}

Conflict of interest SW is an educational consultant and a technological advisory board member of Medtronic Navigation (Louisville, CO, USA). All other authors certify that they have no affiliations with or involvement in any organization or entity with any financial interest (such as honoraria; educational grants; participation in speakers' bureaus; membership, employment, consultancies, stock ownership, or other equity interest; and expert testimony or patent-licensing arrangements) or nonfinancial 
interest (such as personal or professional relationships, affiliations, knowledge, or beliefs) in the subject matter or materials discussed in this manuscript.

Ethical approval All procedures performed in studies involving human participants were in accordance with the ethical standards of the institutional and/or national research committee (Ethics Committee of the Medical University of Vienna) and with the 1964 Helsinki declaration and its later amendments or comparable ethical standards. Informed consent was obtained from all individual participants included in the study.

Abbreviations $C T$, computed tomography; $E M$, electromagnetic; $E O R$, extent of resection; $I C A$, internal carotid artery; $M R$, magnetic resonance; $S O M$, sphenoorbital meningioma; $S O F$, superior orbital fissure; $T M J$, temporomandibular joint; $W H O$, World Health Organization

Open Access This article is distributed under the terms of the Creative Commons Attribution 4.0 International License (http:// creativecommons.org/licenses/by/4.0/), which permits unrestricted use, distribution, and reproduction in any medium, provided you give appropriate credit to the original author(s) and the source, provide a link to the Creative Commons license, and indicate if changes were made.

\section{References}

1. Cannon PS, Rutherford SA, Richardson PL, King A, Leatherbarrow B (2009) The surgical management and outcomes for spheno-orbital meningiomas: a 7-year review of multi-disciplinary practice. Orbit. https://doi.org/10.3109/01676830903104645

2. Pompili A, Derome PJ, Visot A, Guiot G (1982) Hyperostosing meningiomas of the sphenoid ridge-clinical features, surgical therapy, and long-term observations: review of 49 cases. Surg Neurol. https://doi.org/10.1016/S0090-3019(82)80006-2

3. Marcus H, Schwindack C, Santarius T, Mannion R, Kirollos R (2013) Image-guided resection of spheno-orbital skull-base meningiomas with predominant intraosseous component. Acta Neurochir. https://doi.org/10.1007/s00701-013-1662-8

4. Freeman JL, Davern MS, Oushy S, Sillau S, Ormond DR, Youssef AS, Lillehei KO (2017) Spheno-orbital meningiomas: a 16-year surgical experience. World Neurosurg. https://doi.org/10.1016/j. wneu.2016.12.063

5. Shrivastava RK, Sen C, Costantino PD, Della Rocca R (2005) Sphenoorbital meningiomas: surgical limitations and lessons learned in their long-term management. J Neurosurg 103(3):49. https://doi.org/10.3171/jns.2005.103.3.0491

6. Saeed P, Van Furth WR, Tanck M et al (2011) Natural history of spheno-orbital meningiomas. Acta Neurochir. https://doi.org/10. 1007/s00701-010-0878-0

7. Scarone P, Leclerq D, Héran F, Robert G (2009) Long-term results with exophthalmos in a surgical series of 30 sphenoorbital meningiomas. Clinical article. J Neurosurg. https://doi.org/10.3171/2009. 1.JNS081263

8. Bikmaz K, Mrak R, Al-Mefty O (2007) Management of bone-invasive, hyperostotic sphenoid wing meningiomas. J Neurosurg. https://doi.org/10.3171/jns.2007.107.5.905

9. Saeed P, van Furth WR, Tanck M, Freling N, van der Sprenkel JWB, Stalpers LJA, van Overbeeke JJ, Mourits MP (2011) Surgical treatment of sphenoorbital meningiomas. Br J Ophthalmol. https://doi.org/10.1136/bjo.2010.189050

10. Sandalcioglu IE, Gasser T, Mohr C, Stolke D, Wiedemayer H (2005) Spheno-orbital meningiomas: interdisciplinary surgical approach, resectability and long-term results. J Cranio-Maxillofac Surg. https://doi.org/10.1016/j.jcms.2005.01.013
11. Leroy HA, Leroy-Ciocanea CI, Baroncini M, Bourgeois P, Pellerin P, Labreuche J, Duhamel A, Lejeune JP (2016) Internal and external spheno-orbital meningioma varieties: different outcomes and prognoses. Acta Neurochir. https://doi.org/10.1007/s00701-016-2850-0

12. Roser F, Nakamura M, Jacobs C, Vorkapic P, Samii M (2005) Sphenoid wing meningiomas with osseous involvement. Surg Neurol. https://doi.org/10.1016/j.surneu.2004.08.092

13. Jung SH, Ferrer AD, Vela JS, Granados FA. Spheno-orbital meningioma resection and reconstruction: the role of piezosurgery and premolded titanium mesh. https://doi.org/10.1055/s-0031-1286113

14. Maroon JC, Kennerdell JS, Vidovlch DV, Abla A, Sternau L (1994) Recurrent spheno-orbital meningioma. J Neurosurg 80:202-208

15. Li Y, Shi JT, An YZ, Zhang TM, Di Fu J, Zhang JL, Zhao JZ (2009) Sphenoid wing meningioma en plaque: report of 37 cases. Chin Med J. https://doi.org/10.3760/cma.j.issn.0366-6999.2009.20.005

16. Thurnher MM (2009) 2007 World Health Organization classification of tumours of the central nervous system. Cancer Imaging. https://doi.org/10.1007/s00401-007-0243-4

17. Louis DN, Perry A, Reifenberger G, von Deimling A, FigarellaBranger D, Cavenee WK, Ohgaki H, Wiestler OD, Kleihues P, Ellison DW (2016) The 2016 World Health Organization Classification of Tumors of the Central Nervous System: a summary. Acta Neuropathol. https://doi.org/10.1007/s00401-016-1545-1

18. Gonzales M (2000) The 2000 World Health Organization classification of tumours of the nervous system. J Clin Neurosci 8(1):1-3. https://doi.org/10.1054/jocn.2000.0829

19. Mert A, Gan LS, Knosp E, Sutherland GR, Wolfsberger S (2013) Advanced cranial navigation. Neurosurgery $72: 43-53$

20. Terstegge K, Schorner W, Henkes H, Heye N, Hosten N, Lanksch WR (1994) Hyperostosis in meningiomas: MR findings in patients with recurrent meningioma of the sphenoid wings. Am J Neuroradiol 15(3):555-60.

21. Echlin F (1934) Cranial osteomas and hyperostoses produced by meningeal fibroblastomas. Arch Surg 28(2):357-405

22. Globus JH (1937) The meningiomas. Trans Assoc Res Nerv Ment Dis 16:210-265

23. Goyal N, Kakkar A, Sarkar C, Agrawal D (2012) Does bony hyperostosis in intracranial meningioma signify tumor invasion? A radio-pathologic study. Neurol India. https://doi.org/10.4103/ 0028-3886.93589

24. Heick A, Mosdal C, Iorgensen K, Klinken L (1993) Localized cranial hyperostosis of meningioma: a result of neoplastic enzymatic activity. 87(3):243-7.

25. Min J-H, Kang S-H, Lee J-B, Chung Y-G, Lee H-K (2005) Hyperostotic meningioma with minimal tumor invasion into the skull. Neurol Med Chir 45(9):480-3. https://doi.org/10.2176/nmc. 45.480

26. Pieper DR, Al-Mefty O, Hanada Y, Buechner D (1999) Hyperostosis associated with meningioma of the cranial base: secondary changes or tumor invasion. Neurosurgery 44:742-747

27. Salehi F, Jalali S, Alkins R, Il LJ, Lwu S, Burrell K, Gentili F, Croul S, Zadeh G (2013) Proteins involved in regulating bone invasion in skull base meningiomas. Acta Neurochir. https://doi.org/10.1007/ s00701-012-1577-9

28. Zadeh G, Jalali S (2012) Identifying novel pathways involved in regulating bone invasion in meningiomas. Neuro Oncol. https://doi. org/10.1093/neuonc/nos222

29. Akutsu H, Sugita K, Sonobe M, Matsumura A (2004) Parasagittal meningioma en plaque with extracranial extension presenting diffuse massive hyperostosis of the skull. Surg Neurol. https://doi.org/ 10.1016/S0090-3019(03)00521-4

30. Kim KS, Rogers LF, Goldblatt D (1987) CT features of hyperostosing meningioma en plaque. Am J Roentgenol. https:// doi.org/10.2214/ajr.149.5.1017 
31. Bloss HG, Proescholdt MA, Mayer C, Schreyer AG, Brawanski A (2010) Growth pattern analysis of sphenoid wing meningiomas. Acta Neurochir. https://doi.org/10.1007/s00701-009-0556-2

32. Elder JB, Atkinson R, Zee C-S, Chen TC (2007) Primary intraosseous meningioma. Neurosurg Focus. https://doi.org/10. 3171/FOC-07/10/E13

33. Luetjens G, Krauss JK, Brandis A, Nakamura M (2011) Bilateral sphenoorbital hyperostotic meningiomas with proptosis and visual impairment: a therapeutic challenge. Report of three patients and review of the literature. Clin Neurol Neurosurg. https://doi.org/10. 1016/j.clineuro.2011.06.007

34. Schul C, Wassmann H, Skopp GB, Marinov M, Wölfer J, Schuierer G, Joos U, Willich N (1998) Surgical management of intraosseous skull base tumors with aid of operating arm system. Comput Aided Surg 3:312-319

35. Pfisterer WK, Papadopoulos S, Drumm DA, Smith K, Preul MC (2008) Fiducial versus nonfiducial neuronavigation registration assessment and considerations of accuracy. Neurosurgery. https://doi. org/10.1227/01.neu.0000317394.14303.99

36. Stieglitz LH, Fichtner J, Andres R, Schucht P, Krähenbühl AK, Raabe A, Beck J (2013) The silent loss of neuronavigation accuracy: a systematic retrospective analysis of factors influencing the mismatch of frameless stereotactic systems in cranial neurosurgery. Neurosurgery 72:796-807

37. Zhou C, Anschuetz L, Weder S, Xie L, Caversaccio M, Weber S, Williamson T (2016) Surface matching for high-accuracy registration of the lateral skull base. Int J Comput Assist Radiol Surg. https://doi.org/10.1007/s11548-016-1394-3

38. Simas NM, Farias JP (2013) Sphenoid wing en plaque meningiomas: surgical results and recurrence rates. Surg Neurol Int. https:// doi.org/10.4103/2152-7806.114796
39. Schick U, Bleyen J, Bani A, Hassler W (2006) Management of meningiomas en plaque of the sphenoid wing. J Neurosurg. https://doi.org/10.3171/jns.2006.104.2.208

40. Mirone G, Chibbaro S, Schiabello L, Tola S, George B (2009) En plaque sphenoid wing meningiomas: recurrence factors and surgical strategy in a series of 71 patients. Neurosurgery. https://doi.org/ 10.1227/01.NEU.0000345652.19200.D5

41. Ringel F, Cedzich C, Schramm J (2007) Microsurgical technique and results of a series of 63 spheno-orbital meningiomas. Neurosurgery. https://doi.org/10.1227/01.NEU.0000255415. 47937.1A

42. Soyuer S, Chang EL, Selek U, Shi W, Maor MH, DeMonte F (2004) Radiotherapy after surgery for benign cerebral meningioma. Radiother Oncol. https://doi.org/10.1016/j.radonc.2004.01.006

43. Zachenhofer I, Wolfsberger S, Aichholzer M, Bertalanffy A, Roessler K, Kitz K, Knosp E (2006) Gamma-knife radiosurgery for cranial base meningiomas: experience of tumor control, clinical course, and morbidity in a follow-up of more than 8 years. Neurosurgery 58:28-36

44. Talacchi A, De Carlo A, D’Agostino A, Nocini P (2014) Surgical management of ocular symptoms in spheno-orbital meningiomas. Is orbital reconstruction really necessary? Neurosurg Rev. https:// doi.org/10.1007/s10143-014-0517-y

45. Huttner HB, Bergmann O, Salehpour M et al (2018) Meningioma growth dynamics assessed by radiocarbon retrospective birth dating. EBioMedicine. https://doi.org/10.1016/j.ebiom.2017.12.020

Publisher's note Springer Nature remains neutral with regard to jurisdictional claims in published maps and institutional affiliations. 